\title{
ALTERNATIVE APPROACHES TO THE SPECTRAL QUANTITATIVE RESOLUTION OF TWO-COMPONENT MIXTURE BY WAVELET FAMILIES
}

\author{
ERDAL DINÇ ${ }^{I^{*}}$, FAHRETTIN ARSLAN ${ }^{2}$ AND DUMITRU BALEANU ${ }^{3,4}$ \\ ${ }^{1}$ Department of Analytical Chemistry, Faculty of Pharmacy, Ankara University, \\ 06100, Tandoğan, Ankara, Turkey \\ ${ }^{2}$ Department of Statistics, Faculty of Science, Ankara University \\ 06100, Tandoğan, Ankara, Turkey \\ ${ }^{3}$ Department of Mathematics and Computer Sciences, Faculty of Arts and Sciences, Çankaya University, \\ 06530 Balgat, Ankara, Turkey \\ ${ }^{4}$ National Institute for Laser, Plasma and Radiation Physics, Institute of Space Sciences, Magurele-Bucharest, P.O. Box, \\ $M G-23, R 76911$, Romania \\ (Received 26 February 2008 - Accepted 8 September 2008)
}

\begin{abstract}
A new spectral continuous wavelet transform (CWT) methods are proposed for the quantitative analysis of the binary mixtures. The simultaneous spectral resolution of binary mixtures and tablets containing paracetamol (PAR) and chloroxozone (CHL) with overlapping absorption spectra is performed by six wavelet families with no chemical separation procedure. The calibration graphs for the six wavelet families are obtained by the help of the data collected from the CWT- signal amplitudes corresponding to the zero crossing points in the spectral range of $210 \mathrm{~nm}-310 \mathrm{~nm}$. The validation of each wavelet family is carried out by analyzing various synthetic binary mixtures of the above mentioned drugs. The second derivative spectrophotometry (D2) is used to compare the experimental results provided by the analyzed continuous wavelet families and a good coincidence is reported for the proposed analytical approaches.
\end{abstract}

Keywords: Continuous wavelet families; Simultaneous determination; Paracetamol; Chloroxozone; Binary mixture; Tablets.

\section{INTRODUCTION}

In the field of analytical chemistry, various graphical and numerical spectral methods have been applied to the quality control or to the routine analysis of several commercial preparations containing complex mixtures with a constant matrix. The derivative spectrophotometry has been used extensively for the binary mixture analysis. Many reviews have been reported the theoretical basis and applications of derivative spectrophotometry to multicomponent samples ${ }^{1-2}$ however it has several disadvantages, e.g. the signal/noise ratio diminishes in the higher order derivative, the main band and the noise peak may interfere with each other in some cases. In addition the involved parameters (derivative order, smoothing factor, the scale factor and working wavelength) must be well selected for the suitable application of the derivative spectrophotometry. However, the derivative method may not give better results in all cases even if the above conditions are provided. Nowadays, various mathematical calibration models, e.g. classical least squares, inverse least squares, principal component regression and partial least squares have been extensively applied to complex mixture analysis. Classical least squares and inverse least squares have some limited area of analytical applications and for some mixtures the methods can not be applied due to the fact that the matrix effects of samples can not be fully eliminated.

However, principal component regression and partial least squares eliminate the matrix effect but in some cases it requires well knowledge of statistics and involves much time for finding the optimal factor number.

Therefore, it shows us that we need spectral approaches to analyze the complex mixtures.

During the last decades the wavelet transform method became an efficient tool to solve problems in several areas of science and engineering ${ }^{3,4}$. Very recently, the method has been successfully applied to analytical chemistry ${ }^{3-13}$. The advantage of CWT approach over the derivative method is that CWT provides a set of wavelet families which give us a rich resolution of the overlapping absorption spectra. For example, the wavelet transform doesn't require an extra denoising procedure during the spectral transform due to its mathematical algorithm. The use of the spectral CWT provides sharp and higher peak amplitudes possessing low signal-to-noise $(\mathrm{S} / \mathrm{N})$ ratio.

One of the open problem encountered in the application of the continuous wavelet transform (CWT) or the discrete wavelet transform (DWT) in quantitative drug analysis is to find, for a specified mixture, the optimal wavelet family providing the acceptable recovery results. This problem can be handled in principle from the mathematical point of view as an optimal control problem. However, the existence of various wavelet families and the complexity of the spectra create difficulties in handling this problem. The common encountered case is that several families provide the same recoveries for a given complex data system. As a result, we have to analyze the values of other quantities, e.g. mean recovery, the standard deviation and the relative standard deviation in order to decide which wavelet family gives the better recovery results.

The quantitative analysis of PAR combinations with other active compounds by spectrophotometry ${ }^{6,13-24}$ and by HPLC ${ }^{25,26}$ has been obtained in the literature.

The main aim of this study is to develop new spectral alternative resolutions of the mixture PAR and CHL drugs. For the investigated binary mixture, a series of continuous wavelet families (Mexican hat function, Daubechies, Symplets, Coiflets, Biortogonal and Gauss) give us higher recoveries. In order to compare the assay results of six wavelet families, the D2 method is used as a comparison method for the resolution of the above mixture. The validity of six indicated CWT families and DS approaches is performed by analyzing various synthetic mixtures of CHL and PAR compounds. The proposed CWT and DS approaches can be use for the routine quality control of the tablet containing CHL and PAR compounds.

Although the classical derivative spectrophotometry has been applied widely for the resolution of the binary mixtures, in the spectral quantitative analysis this method has several disadvantages and limitations, e.g., only the first-fourth derivative order can be used, it has low sensitivity in ambiguous spectral situation and the decreasing of signal/noise ratio.

Recently, the wavelet method started to play and important role in simultaneous spectral analysis of complex mixtures. The spectral wavelet methods are more useful due to their high sensitivities, self de-noising algorithms, providing sharp peaks together with higher peak amplitudes. The existence of multiple wavelet families is one of the most used advantages in dealing with the alternative spectral transformations which give us different outputs for the quantitative evaluation in mixture analysis.

\section{EXPERIMENTAL}

Apparatus and Software

In this study a Shimadzu UV-1601 double beam UV-Visible spectrophotometer equipped with a fixed slit width $(2 \mathrm{~nm})$ and connected to a computer loaded with Shimadzu UVPC software and a LEXMARK E320 printer was involved to record the absorption spectra and their absorbance measurements. All statistical and signal processing calculations are based on developed algorithms in MATLAB 7.0 and Microsoft EXCEL softwares. 
Commercial tablet formulation

A commercial tablet, namely Parafon ${ }^{\circledR}$ Table produced by SANTA FARMA Pharm. Ind. from Turkey tablet has investigated. Each commercial tablet contains $300 \mathrm{mg}$ PAR and $250 \mathrm{mg}$ CHL compounds.

\section{Standard solutions}

Stock solutions of PAR and CHL have separately prepared by dissolving 50 $\mathrm{mg}$ for each compound in methanol and diluting them into $100-\mathrm{mL}$ calibrated flask. A standard series for each PAR and CHL in the linear concentration range of 4-14 $\mu \mathrm{g} / \mathrm{mL}$ and 6-16 $\mu \mathrm{g} / \mathrm{mL}$, respectively, has obtained by using the above stock solutions. A validation set consisting of 12 synthetic mixture solutions of PAR and CHL in the above concentration range were obtained from stock solutions by the dilution. For all dilutions we have used $0.1 \mathrm{M} \mathrm{HCl}$.

\section{Analysis of commercial tablet}

10 tablets were accurately weighed and powdered in a mortar. An equivalent amount to half tablet containing CHL and PAR was dissolved with methanol to $50 \mathrm{~mL}$ calibrated flask. The content of the flask was mechanically shaken for $30 \mathrm{~min}$. The same content was filtrated with $0.45 \mu \mathrm{m}$ membrane filter and diluted to an appropriate volume with $0.1 \mathrm{~N} \mathrm{HCl}$. The above procedure has repeated 8 times. All proposed spectral methods were applied to the final sample solutions.

\section{METHOD AND THEORETICAL FRAMEWORK}

\section{Wavelets}

Various amounts of wavelet families and functions provide a rich space in which to search for a wavelet which will very efficiently represent a signal in the field of analytical chemistry.

The wavelet function is orthogonal to all functions which are obtained by shifting the wavelet function to right or left by an integer number. More exactly, the wavelet function is orthogonal to all functions which are obtained by dilating the mother by a factor of $2^{j}$ and shifting by multiples of $2^{j}$ units.

It was shown in the literature that the wavelets families have the property to efficiently represent functions possessing localized features. In addition of that, some wavelets may be implemented in an extremely computationally efficient manner by means of a multi-resolution analysis.

Wavelet families and their combinations with other chemometric techniques have been used for the quantitative analysis of complex mixtures.

In the following a briefly fundamental description of a wavelet family is presented. The starting point is to consider a wavelet family $\varnothing(\lambda)(1)$. By scaling and shifting the wavelet function $\varnothing(\lambda)$ a set of functions denoted by $\varnothing \mathrm{a}, \mathrm{b}(\lambda)$ can be obtain as

$$
\Psi_{a, b(\lambda)}=\frac{1}{\sqrt{|a|}} \Psi\left(\frac{\lambda-b}{a}\right) \quad a \neq 0, \quad a, b \in \mathrm{R} .
$$

Here a denotes the scale parameter, $\mathrm{b}$ is the translation parameter and $\mathrm{R}$ denotes the domain of real numbers. A CWT of a given signal $f(\lambda)$ is defined as

$$
\operatorname{CWT}\{\mathrm{f}(\lambda) ; a, b\}=\int_{-\infty}^{\infty} \mathrm{f}(\lambda)^{*} \psi_{a, b}^{*}(\lambda) \mathrm{d} \lambda=\left\langle f(\lambda), \psi_{a, b}\right\rangle,
$$

where the superscript* is the complex conjugate and $\langle f(\ddot{e}) \quad\rangle^{\text {denotes }}$ the inner product of function $\mathrm{f}(\lambda)$ onto the wavelet functiof $f(\ddot{O}) \mathrm{a}, \mathrm{b}(\lambda)$.

In the following we have six wavelet families abbreviated as mex, b2, sym2, coif1, bior4.4, gaus2.

\section{RESULTS AND DISCUSSION}

\section{Alternative spectral CWT approaches}

Figure 1a indicates that the absorption spectra of PAR and CHL overlap in the spectral region $210-320 \mathrm{~nm}$. The existence of the overlapping absorption spectra of the compounds is to render impossible for the spectral simultaneous determination of the PAR and CHL in samples. For this reason, six continuous wavelet families as new alternative spectral methods are proposed for the simultaneous determination of PAR and CHL compounds in their synthetic mixtures and tablets without using any separation step. As it is known, derivative spectrophotometry has been frequently used for the spectral determination of compounds in samples. In all cases, this derivative method may not give desirable results due to overlapping spectra and decrease the signal-to-noise ratio in derivative calculation in binary mixture analysis. It is clear that the use of alternative methods and mathematical approaches for the elimination of the disadvantages of the above mentioned methods needs for the analysis of complex mixtures. Moreover, the alternative spectral CWT approaches can be considered as new examples in the spectral quantitative analysis of two component mixtures.

In this study, several continuous wavelet families for the determination of PAR and CHL are tested and a series of wavelet families, Mexican hat function, Daubechies, Symplets, Coiflets, Biortogonal and Gauss, are suitable for the analysis of the binary mixture of the subject two drugs. The application of six CWT families is given in the following.

\section{CWT calibration graphs}

The absorption spectra of PAR and CHL in the concentration range 4-14 $\mu \mathrm{g} / \mathrm{mL}$ and 6-16 $\mu \mathrm{g} / \mathrm{mL}$ CHL with their tablet samples are plotted in Figure 1a. The absorption spectra consisting of 1024 data points in the wavelength range $210-310 \mathrm{~nm}$, have transferred in the wavelet domain and it was processed by the above mentioned six CWT families. The mexh-CWT, db2-CWT, sym2-CWT, coif1-CWT, bior4.4-CWT and gaus2-CWT spectra have obtained by graphing the CWT- coefficients the provided by Eq. (2) versus the wavelengths, as shown in Figure 1b-g, respectively. At the selected wavelengths, the calibration graphs and their statistical parameters of CHL for each CWT family are indicated in Table 1.The determination of CHL in samples has performed by using the mexh-CWT, db2-CWT, sym2-CWT, coif1-CWT, bior4.4-CWT and gaus2CWT calibration graphs as shown in Table 1. By using a similar procedure as for CHL, the calibration graphs of PAR are depicted in Table 2. The amount of PAR in samples is calculated by using the above calibration graphs.

The red CWT spectra given in Figure 1b-f, correspond to the tablet samples. Transformed spectra of calibration graph and tablet samples have collected in the same Figure in order to demonstrate clearly all transformations of the absorption spectra of two pure compounds an their corresponding tablet samples.

The derivative spectrophotometry has used to verify the six CWT methods. By trying the first and the second derivative we have observed that the second derivative is suitable to check the results of the proposed CWT alternative methods. The results provided by the first derivative method have indicated that this method is not suitable for the quantitative analysis of PAR and CHL.

Figure $1 \mathrm{~h}$ shows the second derivative spectra of CHL and PAR in the linear concentration range of $6-16 \mu \mathrm{g} / \mathrm{mL}$ and $4-14 \mu \mathrm{g} / \mathrm{mL}$, respectively as well as the tablet samples. For all calculations involved in D2 method we used $\Delta \lambda=5$ $\mathrm{nm}$ and a scaling factor of 15 .

The scaling factor was needed in order to increase the peak amplitude. The second derivative spectra was processed by smoothing function with $\Delta \lambda=5$ $\mathrm{nm}$ due to noise elimination procedure. In contrast to derivative method, the spectral CWT approaches do not require the above treatments.

The derivative calibration graphs have obtained by measuring the second derivative amplitudes at 250.9 and $271.8 \mathrm{~nm}$ for CHL and at 262.2.nm for PAR. The linear regression analysis and its statistical results for both compounds are shown in Table 1 and Table 2. The analysis of CHL and PAR in samples is performed by derivative calibration graphs. 

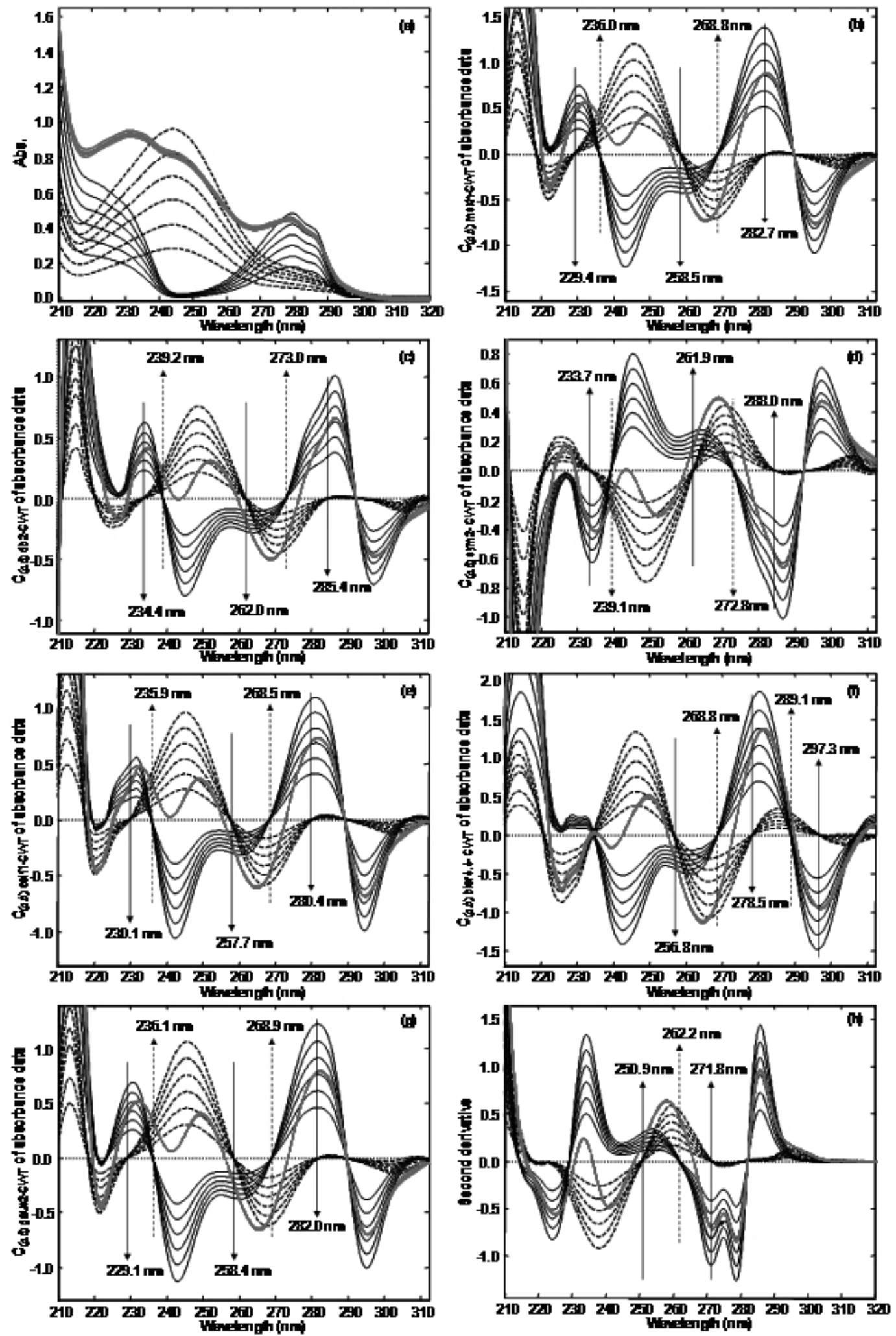

Figure 1. Absorption spectra, b) mexh-CWT spectra, c) db2-CWT spectra, d) sym2-CWT spectra, e) coif1-CWT spectra, f) bior4.4-CWT spectra, g) gaus2CWT spectra and h) D2-spectra of 4,6,8,10,12,14 $\mu \mathrm{g} / \mathrm{mL}$ PAR (-) and 6,8,10,12,14,16 $\mu \mathrm{g} / \mathrm{mL}$ CHL (----) and their tablet samples (-). 
Table 1. CHL linear regression analysis and its corresponding statistical results by the proposed methods

\begin{tabular}{|c|c|c|c|c|c|c|c|c|c|}
\hline CWT & $\lambda(\mathrm{nm})$ & M & $\mathrm{n}$ & $\mathrm{R}$ & $\mathrm{SE}(\mathrm{m})$ & $\mathrm{SE}(\mathrm{n})$ & $\mathrm{SE}(\mathrm{r})$ & $\begin{array}{c}\text { LOD } \\
(\mu \mathrm{g} / \mathrm{mL})\end{array}$ & $\begin{array}{c}\mathrm{LOQ} \\
(\mu \mathrm{g} / \mathrm{mL})\end{array}$ \\
\hline \multirow{3}{*}{ Mexh } & 229.4 & $4.49 \times 10^{-2}$ & $-1.80 \times 10^{-3}$ & 0.9998 & $4.36 \times 10^{-4}$ & $5.02 \times 10^{-3}$ & $3.65 \times 10^{-3}$ & 0.82 & 2.74 \\
\hline & 258.5 & $-2.66 \times 10^{-2}$ & $9.10 \times 10^{-4}$ & 1.0000 & $7.40 \times 10^{-5}$ & $8.53 \times 10^{-4}$ & $6.19 \times 10^{-4}$ & 0.24 & 0.79 \\
\hline & 282.7 & $8.49 \times 10^{-2}$ & $-1.76 \times 10^{-3}$ & 1.0000 & $2.94 \times 10^{-4}$ & $3.39 \times 10^{-3}$ & $2.46 \times 10^{-3}$ & 0.29 & 0.98 \\
\hline \multirow{3}{*}{$\mathrm{db} 2-128$} & 234.4 & $3.87 \times 10^{-2}$ & $-4.75 \times 10^{-4}$ & 0.9998 & $3.39 \times 10^{-4}$ & $3.91 \times 10^{-3}$ & $2.84 \times 10^{-3}$ & 0.74 & 2.47 \\
\hline & 262.0 & $-1.70 \times 10^{-2}$ & $2.26 \times 10^{-3}$ & 0.9999 & $1.28 \times 10^{-4}$ & $1.48 \times 10^{-3}$ & $1.07 \times 10^{-3}$ & 0.58 & 1.94 \\
\hline & 285.4 & $6.05 \times 10^{-2}$ & $-4.33 \times 10^{-4}$ & 1.0000 & $1.65 \times 10^{-4}$ & $1.90 \times 10^{-3}$ & $1.38 \times 10^{-3}$ & 0.23 & 0.77 \\
\hline \multirow{3}{*}{ sym2-128 } & 233.7 & $-3.86 \times 10^{-2}$ & $-1.75 \times 10^{-4}$ & 0.9998 & $3.95 \times 10^{-4}$ & $4.55 \times 10^{-3}$ & $3.30 \times 10^{-3}$ & 0.87 & 2.89 \\
\hline & 261.9 & $1.69 \times 10^{-2}$ & $-2.45 \times 10^{-3}$ & 0.9999 & $1.20 \times 10^{-4}$ & $1.38 \times 10^{-3}$ & $1.00 \times 10^{-3}$ & 0.60 & 2.00 \\
\hline & 288.0 & $-5.90 \times 10^{-2}$ & $-3.06 \times 10^{-3}$ & 0.9999 & $2.96 \times 10^{-4}$ & $3.41 \times 10^{-3}$ & $2.48 \times 10^{-3}$ & 0.42 & 1.42 \\
\hline \multirow{3}{*}{ coif1-144 } & 230.1 & $3.10 \times 10^{-2}$ & $6.42 \times 10^{-4}$ & 0.9997 & $4.02 \times 10^{-4}$ & $4.63 \times 10^{-3}$ & $3.37 \times 10^{-3}$ & 1.10 & 3.67 \\
\hline & 257.7 & $-1.83 \times 10^{-2}$ & $2.01 \times 10^{-3}$ & 1.0000 & $4.22 \times 10^{-5}$ & $4.86 \times 10^{-4}$ & $3.53 \times 10^{-4}$ & 0.19 & 0.65 \\
\hline & 280.4 & $6.78 \times 10^{-2}$ & $-3.01 \times 10^{-3}$ & 0.9999 & $5.01 \times 10^{-4}$ & $5.77 \times 10^{-3}$ & $4.19 \times 10^{-3}$ & 0.63 & 2.09 \\
\hline \multirow{3}{*}{ bior4.4-224 } & 256.8 & $-2.70 \times 10^{-2}$ & $7.15 \times 10^{-4}$ & 0.9999 & $1.76 \times 10^{-4}$ & $2.03 \times 10^{-3}$ & $1.47 \times 10^{-3}$ & 0.55 & 1.84 \\
\hline & 278.5 & $1.09 \times 10^{-1}$ & $5.24 \times 10^{-3}$ & 1.0000 & $4.85 \times 10^{-4}$ & $5.59 \times 10^{-3}$ & $4.06 \times 10^{-3}$ & 0.38 & 1.26 \\
\hline & 297.3 & $-9.12 \times 10^{-2}$ & $-4.83 \times 10^{-3}$ & 1.0000 & $2.74 \times 10^{-4}$ & $3.16 \times 10^{-3}$ & $2.29 \times 10^{-3}$ & 0.25 & 0.85 \\
\hline \multirow{3}{*}{ gaus2-64 } & 229.1 & $4.06 \times 10^{-2}$ & $-8.78 \times 10^{-4}$ & 0.9998 & $4.18 \times 10^{-4}$ & $4.82 \times 10^{-3}$ & $3.50 \times 10^{-3}$ & 0.87 & 2.91 \\
\hline & 258.4 & $-2.32 \times 10^{-2}$ & $1.75 \times 10^{-3}$ & 1.0000 & $6.20 \times 10^{-5}$ & $7.14 \times 10^{-4}$ & $5.19 \times 10^{-4}$ & 0.23 & 0.76 \\
\hline & 282.0 & $7.66 \times 10^{-2}$ & $-2.58 \times 10^{-3}$ & 1.0000 & $3.48 \times 10^{-4}$ & $4.01 \times 10^{-3}$ & $2.92 \times 10^{-3}$ & 0.38 & 1.28 \\
\hline \multirow{2}{*}{ D2 } & 250.9 & $1.89 \times 10^{-2}$ & $-5.53 \times 10^{-3}$ & 0.9998 & $2.10 \times 10^{-4}$ & $2.42 \times 10^{-3}$ & $1.76 \times 10^{-3}$ & 0.86 & 2.87 \\
\hline & 271.8 & $-6.91 \times 10^{-2}$ & $7.70 \times 10^{-3}$ & 0.9997 & $8.86 \times 10^{-4}$ & $1.02 \times 10^{-2}$ & $7.41 \times 10^{-3}$ & 0.99 & 3.30 \\
\hline
\end{tabular}

$r=$ Correlation coefficient of the regression function, $m=$ Slope of the regression function, $n=$ Intercept of the regression function, $r(S E)=S t a n d a r d$ error of the correlation coefficient, $\mathrm{m}(\mathrm{SE})=$ Standard error of the slope, $\mathrm{n}(\mathrm{SE})=$ Standard error of the intercept, LOD $=$ Limit of detection, LOQ $=$ Limit of quantitation

Validation of spectral CWT methods

In the concentration range of 6-16 $\mu \mathrm{g} / \mathrm{mL}$ for CHL and 4-14 $\mu \mathrm{g} / \mathrm{mL}$ for PAR, the six spectral CWT approaches have provided good linearities as indicated by the correlation coefficients ( $\mathrm{r}$ ) shown in Table 1 and Table 2, respectively. Also a good linearity for CHL and PAR calibration graphs has reported for D2 method in the above concentration range (see Table 1 and Table 2).

Table 2. PAR linear regression analysis and its corresponding statistical results by the proposed methods

\begin{tabular}{|c|c|c|c|c|c|c|c|c|c|}
\hline Method & $\lambda .(\mathrm{nm})$ & $\mathrm{m}$ & $\mathrm{n}$ & $\mathrm{r}$ & $\mathrm{SE}(\mathrm{m})$ & $\mathrm{SE}(\mathrm{n})$ & $\mathrm{SE}(\mathrm{r})$ & LOD & LOQ \\
\hline \multirow{3}{*}{ mexh } & 236.0 & $3.51 \times 10^{-2}$ & $-5.23 \times 10^{-3}$ & 0.9993 & $6.38 \times 10^{-4}$ & $6.14 \times 10^{-3}$ & $5.34 \times 10^{-3}$ & 1.29 & 4.29 \\
\cline { 2 - 10 } & 268.8 & $-4.92 \times 10^{-2}$ & $1.98 \times 10^{-4}$ & 0.9999 & $3.76 \times 10^{-4}$ & $3.62 \times 10^{-3}$ & $3.15 \times 10^{-3}$ & 0.49 & 1.64 \\
\hline \multirow{3}{*}{$\mathrm{db} 2-128$} & 239.2 & $1.83 \times 10^{-2}$ & $-4.88 \times 10^{-3}$ & 0.9994 & $3.14 \times 10^{-4}$ & $3.02 \times 10^{-3}$ & $2.63 \times 10^{-3}$ & 1.21 & 4.04 \\
\cline { 2 - 10 } & 273.0 & $-2.99 \times 10^{-2}$ & $9.73 \times 10^{-4}$ & 0.9999 & $2.30 \times 10^{-4}$ & $2.21 \times 10^{-3}$ & $1.92 \times 10^{-3}$ & 0.54 & 1.81 \\
\hline \multirow{3}{*}{ sym2-128 } & 239.1 & $-1.80 \times 10^{-2}$ & $6.87 \times 10^{-3}$ & 0.9994 & $3.02 \times 10^{-4}$ & $2.91 \times 10^{-3}$ & $2.53 \times 10^{-3}$ & 1.19 & 3.96 \\
\cline { 2 - 11 } & 272.8 & $3.02 \times 10^{-2}$ & $-7.94 \times 10^{-4}$ & 0.9999 & $2.35 \times 10^{-4}$ & $2.27 \times 10^{-3}$ & $1.97 \times 10-^{3}$ & 0.55 & 1.83 \\
\hline \multirow{3}{*}{ coif1-144 } & 235.9 & $2.92 \times 10^{-2}$ & $-9.13 \times 10^{-3}$ & 0.9996 & $4.33 \times 10^{-4}$ & $4.17 \times 10^{-3}$ & $3.62 \times 10^{-3}$ & 1.05 & 3.50 \\
\cline { 2 - 11 } & 268.5 & $-4.01 \times 10^{-2}$ & $-6.19 \times 10^{-5}$ & 0.9999 & $3.45 \times 10^{-4}$ & $3.32 \times 10^{-3}$ & $2.89 \times 10^{-3}$ & 0.61 & 2.03 \\
\hline \multirow{3}{*}{ bior4.4-224 } & 268.8 & $-7.21 \times 10^{-2}$ & $3.42 \times 10^{-3}$ & 0.9999 & $5.79 \times 10^{-4}$ & $5.57 \times 10^{-3}$ & $4.84 \times 10^{-3}$ & 0.57 & 1.89 \\
\cline { 2 - 11 } & 289.1 & $2.12 \times 10^{-2}$ & $-1.46 \times 10^{-2}$ & 0.9997 & $2.59 \times 10^{-4}$ & $2.49 \times 10^{-3}$ & $2.16 \times 10^{-3}$ & 0.86 & 2.87 \\
\hline \multirow{2}{*}{ gaus2-64 } & 236.1 & $3.07 \times 10^{-2}$ & $-8.50 \times 10^{-3}$ & 0.9995 & $4.92 \times 10^{-4}$ & $4.73 \times 10^{-3}$ & $4.11 \times 10^{-3}$ & 1.13 & 3.78 \\
\cline { 2 - 11 } & 268.9 & $-4.38 \times 10^{-2}$ & $3.18 \times 10^{-4}$ & 0.9999 & $3.37 \times 10^{-4}$ & $3.24 \times 10^{-3}$ & $2.82 \times 10^{-3}$ & 0.54 & 1.81 \\
\hline \multirow{2}{*}{ D2 } & 262.2 & $3.72 \times 10^{-2}$ & $1.79 \times 10^{-3}$ & 0.9998 & $3.42 \times 10^{-4}$ & $3.29 \times 10^{-3}$ & $2.86 \times 10^{-3}$ & 0.59 & 1.98 \\
\hline
\end{tabular}

The proposed spectral mexh-CWT, db2-CWT, sym2-CWT, coif1-CWT, bior4.4-CWT and gaus2-CWT methods have tested by considering 12 different mixtures consisting of CHL and PAR within the working concentration range. D2 method has applied to the above mixtures.

It was observed that all spectral approaches provided successful accuracies and precisions according to the obtained mean recoveries and the relative standard deviations indicated in Table 3 for CHL and in Table 4 for PAR. The numerical values of the mean recoveries show that gauss2-CWT gives $99.9 \%$ for CHL and mexh-CWT approaches to $100 \%$ for PAR (see Table 3 and Table 4). By reading the numerical values of the lowest relative standard deviations (RSD) we conclude that sym2-CWT gives $1.12 \%$ for CHL and 0.50 for PAR as illustrated in Table 3 and Table 4. 


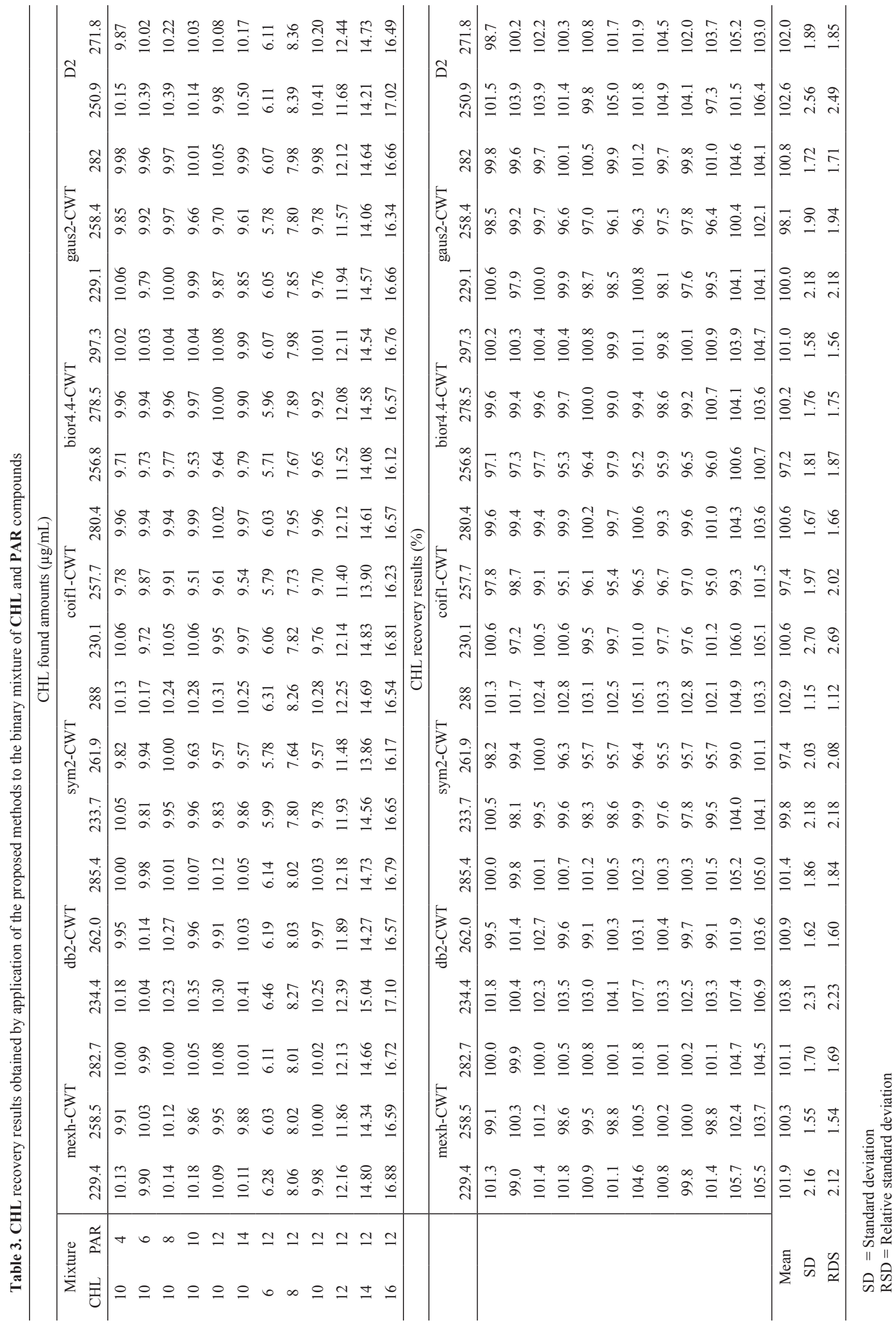




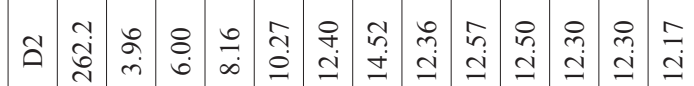

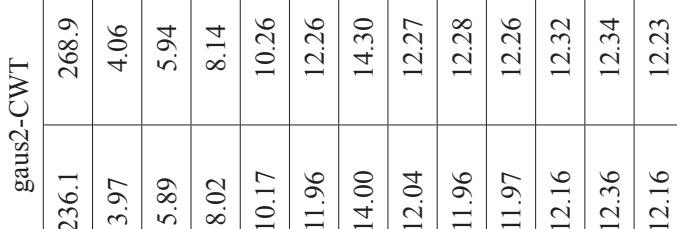

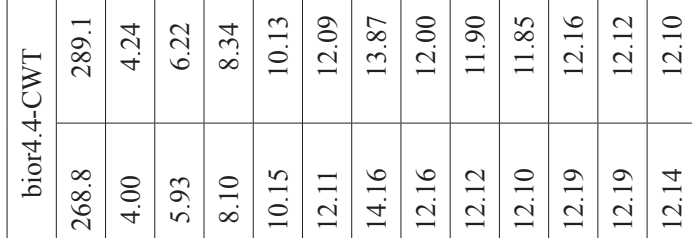

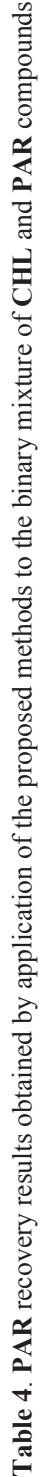

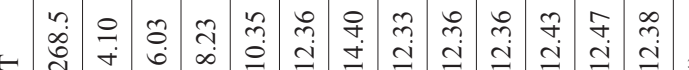

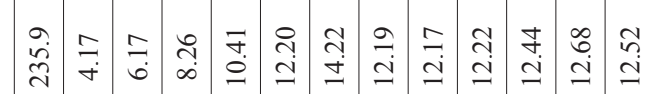

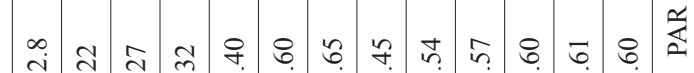

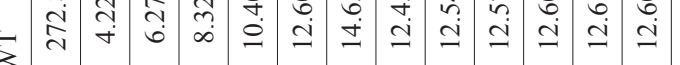

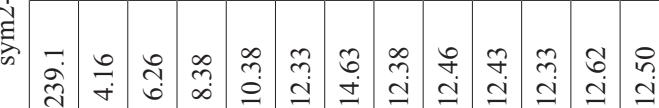

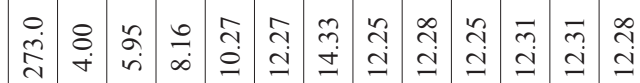

年

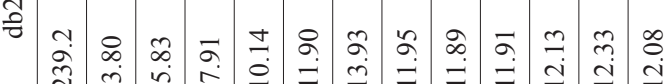

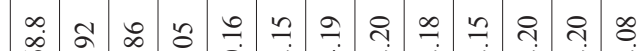

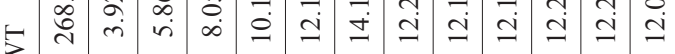

己

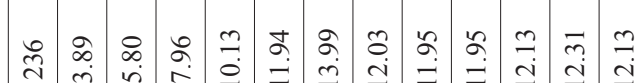

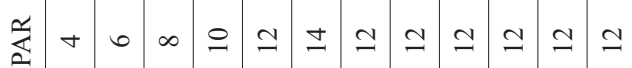

竞

빈으으으으 $0 \infty$ 의

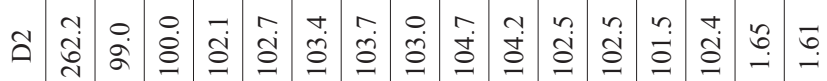

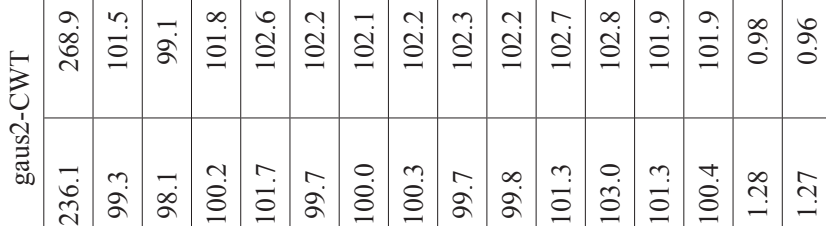

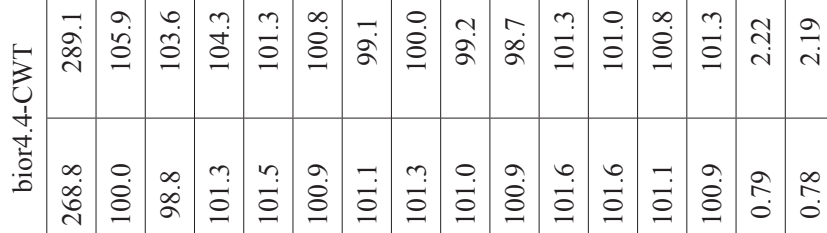

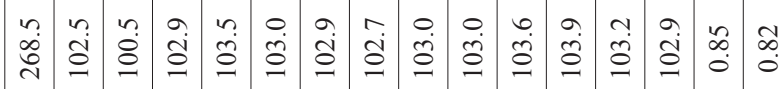

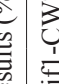

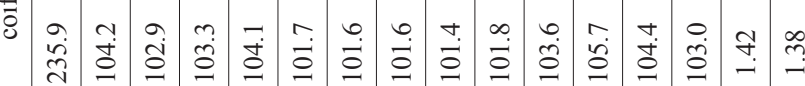

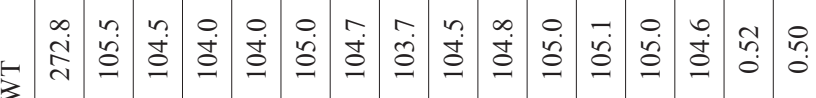

บุ

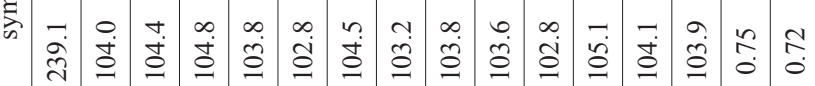

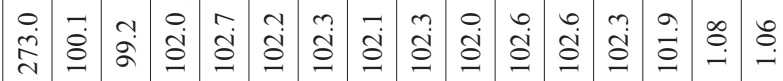

לุin

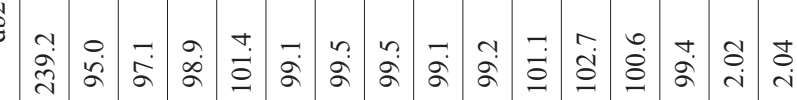

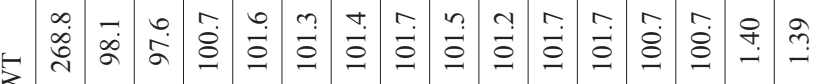

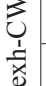

থ) 


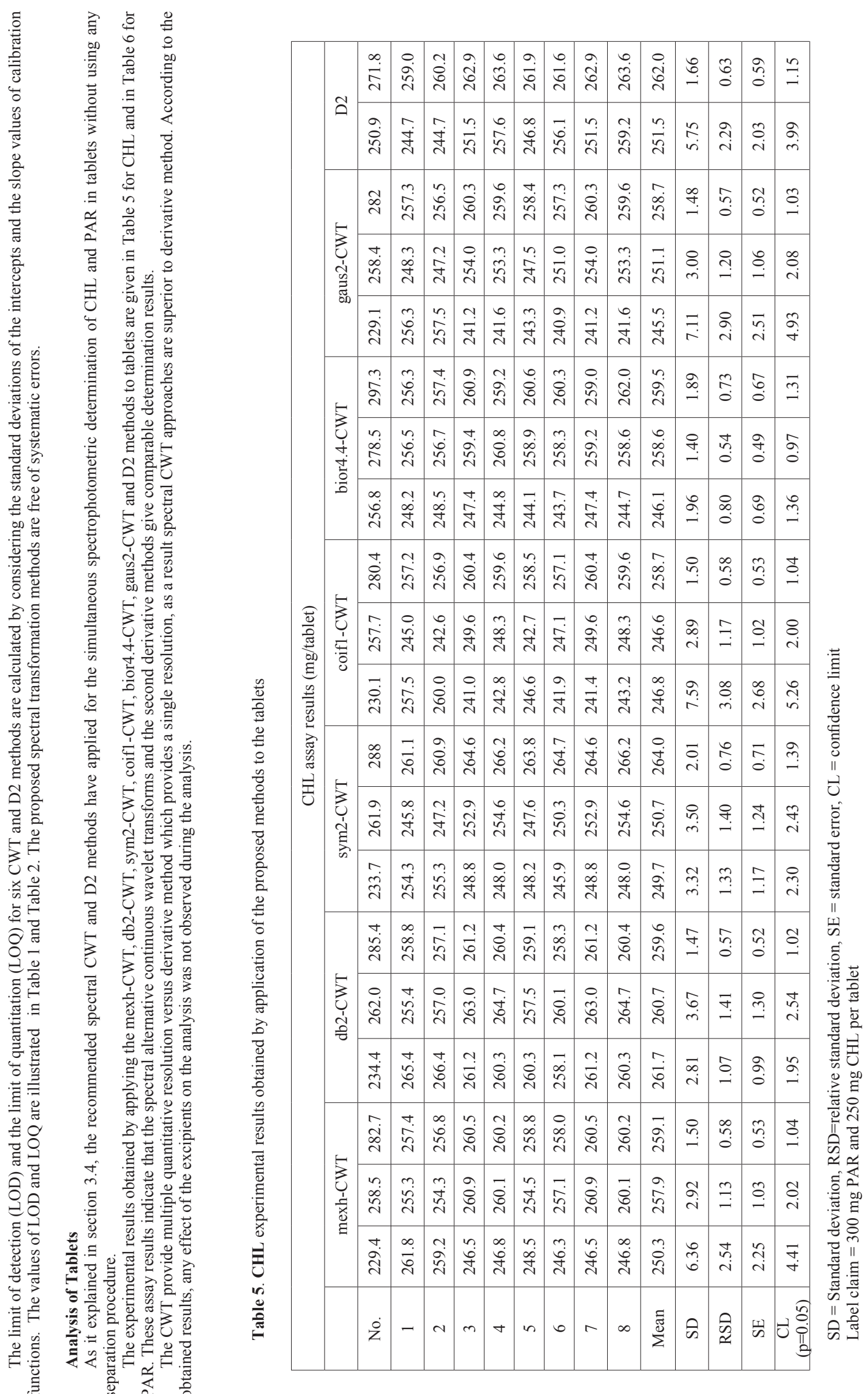




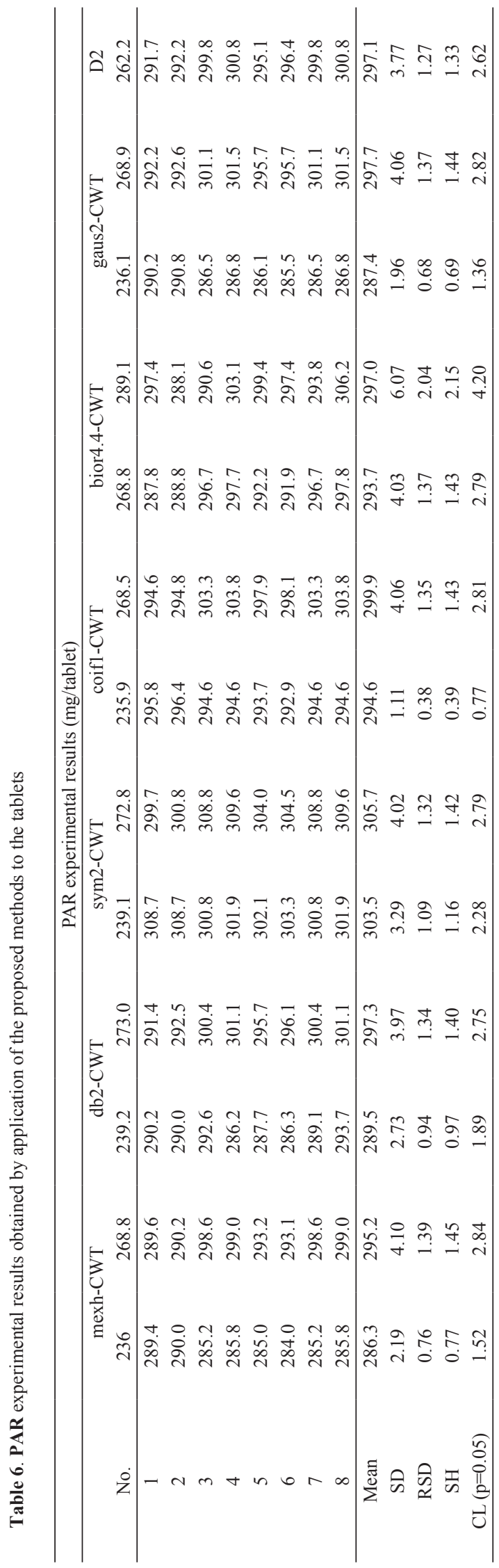

\section{CONCLUSION}

The study illustrated a successful application of the spectral CWT approaches to the analysis of synthetic binary mixtures as well as tablets containing CHL and PAR compounds. A series of six CWT families give satisfactory determination results despite the overlapping absorption spectra of the related compounds in the same spectral range of $210-320 \mathrm{~nm}$. The results of this manuscript show that the role of the CWT method becomes very important for the rapid, accurate, precise, very cheap and easy to apply of the quantitative analysis of two-component mixtures. The proposed CWT approaches do not need the extra treatments as in the case of the classical derivative method. Therefore, the proposed spectral alternative CWT processing method can replace successfully the classical derivative method. Besides, the presence of several continuous wavelet families providing desirable results open new problem in the field of chemometrics, namely the optimal control like problem for finding the optimal CWT family for a given complex mixture.

Finally, we strongly believe that this alternative approaches can successfully used in routine analysis and quality control of tablets containing the related compounds.

\section{REFERENCES}

1. J. Karpinska, Talanta $64,801,(2004)$.

2. C. O. Bosch, F. R.Sanchez, Anal. Chim. 518 ,1,(2004).

3. B. Walczak, Wavelets in Chemistry, Elsevier Press, Amsterdam, The Netherlands, 2000

4. I. Daubechies, Ten Lectures on Wavelets, Society for Industrial and Applied Mathematics, Philadelphia, 1992.

5. E. Dinç, D. Baleanu, A review on the wavelet transform applications in analytical chemistry. Mathematical Methods in Engineering, eds. K.Taş, J.A. Tenreiro Machado, D. Baleanu. Springer, The Netherlands, 2007; pp. 265-284.

6. E. Dinç, D. Baleanu, Rev. Chim. (Bucuresti) 56, 937, (2005).

7. E. Dinç, D. Baleanu, Talanta 59, 707, (2003).

8. E. Dinç, D. Baleanu, J. Pharm. Biomed. Anal 31, 969, (2003).

9. E. Dinç, D. Baleanu, J. AOAC Int. 87(2), 360, (2004).

10. E. Dinç, D. Baleanu, J. AOAC Int. 87(4), 834, (2004).

11. E. Dinc, S. Kaya, T. Doganay, D. Baleanu, J. Pharm. Biomed. Anal. 44, 991, (2007).

12. E. Dinç, D. Baleanu, O. Ustundag, Spectr. Lett. 36, 341, (2003).

13. P. L. Lopez-de-Alba, L. Lopez-Martinez, L. I. Michelinni-Rodriguez, K. Wrobel, J. Amador-Hernandez, Analyst, 122, 1575, (1997).

14. L. Lopez-Martinez, P. L. Lopez-de-Alba, L. Manuele de-Leon-Rodriguez, M. L. Yepez-Murietta, J. Pharm. Biomed. Anal. 30, 77, (2002).

15. E. Dinç, J. Pharm. Biomed. Anal. 33, 605, (2003).

16. C. K. Markopoulou, J. E. Koundourelllis, J. Pharm. Biomed. Anal. 33 , 1163, (2003)

17. O. Üstundag, E. Dinç, Pharmazie 58, 623, (2003).

18. E. Dinc, F. Onur, Analusis, 25, 55, (1997).

19. E. Dinç, M. Kanbur, J. Pharm. Biomed. Anal. 28, 779, (2002).

20. E. Dinç, G. Ragno, E. Iole, D. Baleanu, J. AOAC Int. 89, 1538, (2006).

21. E. Dinç, D. Baleanu, Spectr. Acta Part A 63, 631, (2006).

22. E. Dinç, A. Ozdemir, F. Onur, D. Baleanu, H. Aksoy, C. Yucesoy, Rev. Chim (Bucuresti) 57, 368, (2006).

23. E. Dinç, A. Ozdemir, H. Aksoy, D. Baleanu, J. Liq. Chrom. Rel. Tech. 29, 1803, (2006).

24. R. T. Sane, M. Gadgil, J. Plan. Chromat. 15, 76, (2002).

25. A. R. Khan, M. J. Akhtar, R. Mahmood, S. M. Ahmed, S. Malook, M. Iqbal, J. Pharm. Biomed. Anal. 22, 111, (2000).

26. S. C.Su, H.H. Chou, P. C. Chang, C. H. Liu, S. S . Chou, J. Food Drug Anal. 12, 244, (2004). 